\title{
Effective Treatment of Adult Parasomnias with Keishikaryukotsuboreito in Four Cases
}

\author{
Mayumi Tamada ${ }^{1,2}$, Shinji Ueno ${ }^{1}$, Kako Watanabe ${ }^{2}$ and Shin-ichi Muramatsu ${ }^{1,3}$
}

\begin{abstract}
:
Parasomnias are undesirable behaviors or experiences during sleep that manifest clinically as abnormal behavior, emotions, and nightmares. We herein report four elderly parasomnia patients who were successfully treated for abnormal nocturnal behaviors, including rapid eye movement (REM) sleep behavior disorders, with Keishikaryukotsuboreito (KRB), a Japanese traditional herbal medicine. KRB resolved nocturnal violent behaviors and sleep walking without any adverse effects. In one patient, occipital dominant spike-wave complexes induced by $3-\mathrm{Hz}$ photic stimulation were reduced after KRB treatment, suggesting that KRB has inhibitory effects on brain irritability. KRB may represent a safe therapeutic option for treating parasomnias in the elderly.
\end{abstract}

Key words: Keisikaryukotsuboreito, parasomnia, REM sleep behavior disorder

(Intern Med Advance Publication)

(DOI: 10.2169/internalmedicine.7952-21)

\section{Introduction}

Parasomnias are defined as undesirable behaviors or experiences that occur during entry into sleep, within sleep, or when arousing from sleep (1). Parasomnias can be divided into two main classes: those that occur during rapid eye movement (REM) sleep, and those that occur in non-REM (NREM) sleep. For example, during sleep, fluctuations in the sleep-wake cycle can cause instability in the sleeper's consciousness, and whether they are in a sleeping state or a waking state becomes ambiguous, leading to parasomnia. Parasomnias can also occur during uninvolved sleep states (2). The combination of one or more of these states results in an unstable state of altered consciousness, manifesting as a parasomnia $(3,4)$.

REM sleep behavior disorder (RBD) is often associated with REM-related dream content and is characterized by atonia and abnormal behaviors, such as shouting, screaming, or acts of violence. Dreams in RBD are usually vivid and vigorous and therein can induce patients to be violent $(5,6)$. Previously, RBD was considered a rare parasomnia. However, idiopathic RBD has been recognized as a prodromal feature of $\alpha$-synucleopathies, such as Parkinson's disease or Lewy body disease, and is therefore more common that previously realized $(5,7,8)$. Consequently, RBD is now recognized as an early and specific indicator of $\alpha$-synucleinrelated neurodegeneration (9). In contrast, NREM sleep parasomnia is characterized by arousal disorders, including sleep walking, sleep terrors, and confusional arousals. In addition, sleep-related eating disorders and unusual sexual behaviors may also be present (10).

Sleep parasomnia-associated behavior during REM or NREM sleep disrupts sleep and consequently negatively impacts one's health. In particular, violent nocturnal abnormal behaviors can cause serious injuries, not only to patients but also to their bed partners. Thus, parasomnias in adults require appropriate therapeutic intervention.

Although benzodiazepines or anticonvulsants, such as clonazepam, are used to treat parasomnia patients, their efficacy remains unclear. In addition, the long-term use of various psychotropic drugs can sometimes induce adverse effects, including daytime drowsiness, cognitive impairment, or disorder of equilibrium $(10,11)$. These adverse effects are particularly problematic for elderly patients, who are prone to falling or may already have mild cognitive impairment.

${ }^{1}$ Division of Oriental Medicine, Center for Community Medicine, Jichi Medical University, Japan, ${ }^{2}$ Azabu Muse Clinic, Japan and ${ }^{3}$ Division of Neurology, Department of Medicine, Jichi Medical University, Japan

Received: May 16, 2021; Accepted: August 29, 2021; Advance Publication by J-STAGE: October 19, 2021

Correspondence to Dr. Shin-ichi Muramatsu, muramats@jichi.ac.jp 
Table 1. Patients' Baseline Characteristics.

\begin{tabular}{|c|c|c|c|c|c|}
\hline Subject & $\begin{array}{l}\text { Age } \\
\text { (years) }\end{array}$ & Sex & Underlining condition ${ }^{1}$ & Concomitant medication ${ }^{2}$ & Laboratory findings \\
\hline 1 & 72 & M & Parkinson's disease & Levodopa equivalent & $\begin{array}{l}\text { REM sleep without atonia on vPSG } \\
\text { Reduced uptake on MIBG scintigraphy } \\
\text { and FMT-PET }\end{array}$ \\
\hline 2 & 74 & $\mathrm{~F}$ & Mild brain ischemia & Cilostazol & $\begin{array}{l}\text { Spike-wave complexes induced with } \\
3 \mathrm{~Hz} \text { photic stimulation on EEG }\end{array}$ \\
\hline 3 & 73 & M & Mild hypertension & Losartan & No epileptic discharge on EEG \\
\hline 4 & 77 & M & $\begin{array}{l}\text { Diabetes mellitus } \\
\text { Abdominal aneurysm }\end{array}$ & Glimepiride Luseogliflozin & $\mathrm{HbA} 1_{\mathrm{c}} 8.4 \%$ eGFR $41 \mathrm{~mL} / \mathrm{min} / 1.73 \mathrm{~m}^{2}$ \\
\hline
\end{tabular}

EEG: electroencephalography, eGFR: estimated glomerular filtration rate, F: female, FMT-PET: positron emission tomography with 6- $\left[{ }^{18} \mathrm{~F}\right]$ fluoro-L- $m$-tyrosine tracer, M: male, MIBG: metaiodobenzylguanidine myocardial scintigraphy, vPSG: video polysomnography

${ }^{1}$ None of the patients had dementia. ${ }^{2}$ No antipsychotic drugs were prescribed.

Table 2. Clinical Outcomes Following KRB Administration.

\begin{tabular}{|c|c|c|c|}
\hline Subject & $\begin{array}{l}\text { Characteristics of abnormal behavior before treatment }{ }^{1} \\
\text {-Period of the time since the abnormal behavior start } \\
\text {-Frequency of the abnormal behavior }\end{array}$ & $\begin{array}{l}\text { Days taken to alleviate } \\
\text { the abnormal behavior }\end{array}$ & $\begin{array}{l}\text { Observation period }{ }^{2} \\
\text { (months) }\end{array}$ \\
\hline \multirow[t]{2}{*}{1} & Visual hallucinations during the daytime & & \\
\hline & $\begin{array}{l}\text { Dream-enacting behavior, including shouting, and hitting motions } \\
\cdot 6 \text { months } \\
\cdot 3 \text { times or above per weeks }\end{array}$ & 2 & 6 \\
\hline \multirow[t]{2}{*}{2} & Talk and walked during sleep & & \\
\hline & $\begin{array}{l}\text { Nightmare } \\
\cdot 3 \text { months } \\
\cdot 1-2 \text { times per week }\end{array}$ & 2 & 6 \\
\hline \multirow[t]{2}{*}{3} & Dream-enacting behavior, including shouting, and hitting motions & & \\
\hline & $\begin{array}{l}\text { Nightmare } \\
\cdot 2 \text { months } \\
\cdot 1-2 \text { times per week }\end{array}$ & 4 & 6 \\
\hline 4 & $\begin{array}{l}\text { Dream-enacting behavior, including shouting, and hitting motions } \\
\text { Nightmare accompanied by autonomic responses } \\
\cdot 3 \text { months } \\
\cdot 1-2 \text { times per week }\end{array}$ & 3 & 24 \\
\hline
\end{tabular}

${ }^{1}$ No patient had a prior history of sleep-related abnormal behaviors. ${ }^{2}$ No recurrence was observed during this period.

There is thus an urgent need to establish effective and sideeffect-free treatments.

In Japanese traditional medicine, certain herbal medicines, including Yokukansan (YKS) and Keishikaryukotsuboreito (KRB), are used to treat a variety of sleep disorders. In particular, KRB has been indicated for insomnia, oversensitivity, nervous imbalance, night sweating, and sexual deviation in elderly individuals (12).

We herein report four elderly patients whose parasomnias were effectively treated with KRB without any adverse effects.

\section{Methods}

Patients 1,2, and 3 took $7.5 \mathrm{~g} /$ daily KRB extract granules orally divided across 3 separate administrations prior to meals. Patient 4 took $2.5 \mathrm{~g}$ of the drug before going to bed at night. The $7.5 \mathrm{~g}$ of KRB extract granules (Product code;
TJ-26, Tsumura \& Co. Tokyo, Japan) contained $3.25 \mathrm{~g}$ of a dried extract comprised of 7 crude drugs: cinnamon bark 4.0 $\mathrm{g}$, peony root $4.0 \mathrm{~g}$, jujube $4.0 \mathrm{~g}$, oyster shell $3.0 \mathrm{~g}$, longgu $3.0 \mathrm{~g}$, glycyrrhiza $2.0 \mathrm{~g}$, ginger $1.5 \mathrm{~g}$. KRB and these seven crude drugs are listed in the Japanese Pharmacopeia (13).

\section{Case Presentations}

Patients' characteristics and clinical courses are summarized in Table and 2, respectively.

\section{- Patient 1}

A 72-year-old man had been diagnosed with rigidhypokinetic Parkinson's disease 5 years ago. Six months before his presentation, he complained of visual hallucinations during the daytime, such as that dwarves were talking to each other. His wife further reported that he experienced frequent episodes of dream-enacting behavior, including shouting and hitting motions associated with vivid dreams.

${ }^{123}$ Iodine-MIBG myocardial scintigraphy showed that the 


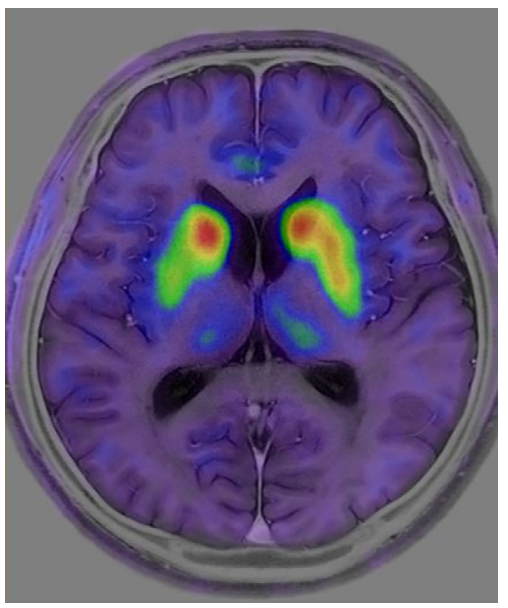

Figure 1. Positron emission tomography of Patient 1. The uptake of $6-\left[{ }^{18} \mathrm{~F}\right]$ fluoro-L-m-tyrosine, a tracer for aromatic $\mathrm{L}$ amino acid decarboxylase, was asymmetrically reduced in the putamen, mostly on the right side of the image.

heart-to-mediastinum $(\mathrm{H} / \mathrm{M})$ ratio was 1.71 in the early phase and 1.45 in the late phase (normal H/M uptake ratio: $\geq 2.2$ ), indicating a reduced cardiac uptake. Positron emission tomography (PET) demonstrated that the uptake of the aromatic 1-amino acid decarboxylase tracer 6- $\left[{ }^{18} \mathrm{~F}\right]$ fluoro-1- $m$ tyrosine was reduced in the putamen, suggesting dopaminergic hypofunction (Fig. 1) (14). He had been receiving 400 $\mathrm{mg}$ of levodopa equivalent daily doses for his Parkinson's disease, which controlled his motor symptoms well. His cognitive function was normal, scoring 30 points on a minimental state examination (MMSE). We confirmed the presence of REM sleep without atonia (RWA) on video polysomnography (vPSG) and diagnosed his abnormal behaviors as RBD.

Two days after starting KRB (7.5 g/day), his visual hallucination and dream-enacting behaviors disappeared. He continued to take KRB without changing his other medications for six months, and his parasomnia did not recur.

- Patient 2

A 74-year-old woman visited our hospital because of a buttocks bruise. One week after moving to her newly built house three months prior to her presentation, she began to frequently talk during her sleep. She reported that she had not experienced abnormal sleep behavior in her childhood. During a recent episode, she walked during her sleep, fell down, and consequently bruised her buttocks. She also suffered from terrible nightmares of a large snake.

Her MMSE score was 26, with 4 points deducted in the calculation test. Her neurological examination findings were normal, while brain magnetic resonance imaging (MRI) revealed small ischemic lesions in her cerebral white matter. She took $100 \mathrm{mg}$ of cilostazol daily. The patient's laboratory testing showed normal blood chemistry. On electroencephalography (EEG), occipital dominant spike-wave complexes were induced with $3-\mathrm{Hz}$ photic stimulation, and as a result, she was diagnosed with a photosensitive constitution
(Fig. 2)

She was able to recall vivid dreams, and she would seemingly talk in her sleep. Since walking during RBDs is considered quite uncommon, we provisionally diagnosed her with a parasomnia overlap disorder in which both probable RBD and arousal disorder symptoms had manifested concurrently $(15,16)$. We prescribed her KRB (7.5 g/day), and after 2 days, her abnormal sleep behaviors resolved, and she no longer experienced nightmares for 6 months. Interestingly, after receiving medication, the frequency and magnitude of spike-wave complexes induced by $3-\mathrm{Hz}$ photic stimulation decreased (Fig. 3).

- Patient 3

A 73-year-old man presented to our clinic complaining of frequent nightmares and abnormal sleep behaviors, which had commenced 2 months before his first visit. In his sleep, he had shouted to his wife, "I'll kill you", hit her, and tried to strangle her. Upon arousal, he had explained that he was fighting a robber in his dream. He complained of occasional forgetfulness but was independent in his daily life and had no obvious dementia. Neurological examinations and brain MRI findings were normal. He had been taking $25 \mathrm{mg}$ of losartan daily for mild hypertension since he was 65 years old. His blood testing showed no specific abnormalities.

We provisionally diagnosed his parasomnia as a probable RBD. Four days after taking KRB (7.5 g/day), his abnormal sleep behaviors disappeared. His wife and he were once more able to sleep soundly after the medication. He continued to take KRB for six months, and his parasomnia did not recur.

\section{- Patient 4}

A 77-year-old man visited our hospital complaining of sleep disturbance. A detailed interview revealed that each night he screamed loudly during sleep because of nightmares, such as the belief that his ceiling was infested with insects or that he was being attacked by a samurai, an ancient Japanese soldier. In addition to screaming loudly, he often showed gestures that he was shaking off insects or fighting someone while sleeping. His screams were often accompanied by autonomic responses, such as sweating. He was independent in his daily life, and there was no evidence of dementia.

Since he was being treated for diabetes, his abnormal sleep behaviors were initially suspected to be due to hypoglycemia. However, his $\mathrm{HbA} 1_{c}$ was $8.4 \%$ (normal value, $4.9 \%$ to $6.0 \%$ ), and he had never reported a hypoglycemic episode. He had been taking $0.5 \mathrm{mg}$ of glimepiride and 2.5 $\mathrm{mg}$ of luseogliflozin hydrate daily for diabetes. He was not taking any antipsychotic drugs. Abdominal CT showed an aortic aneurysm but no evidence of a pancreatic tumor.

Adjusting his diabetes treatment did not improve his symptoms. We subsequently treated his nocturnal abnormal behaviors with a prescription for $2.5 \mathrm{~g} /$ day of $\mathrm{KRB}$ to be taken before going to bed at night. We started at the minimum dose of KRB, as he had a decreased renal function, indicated by a blood creatinine level of $1.32 \mathrm{mg} / \mathrm{dL}$ (normal 


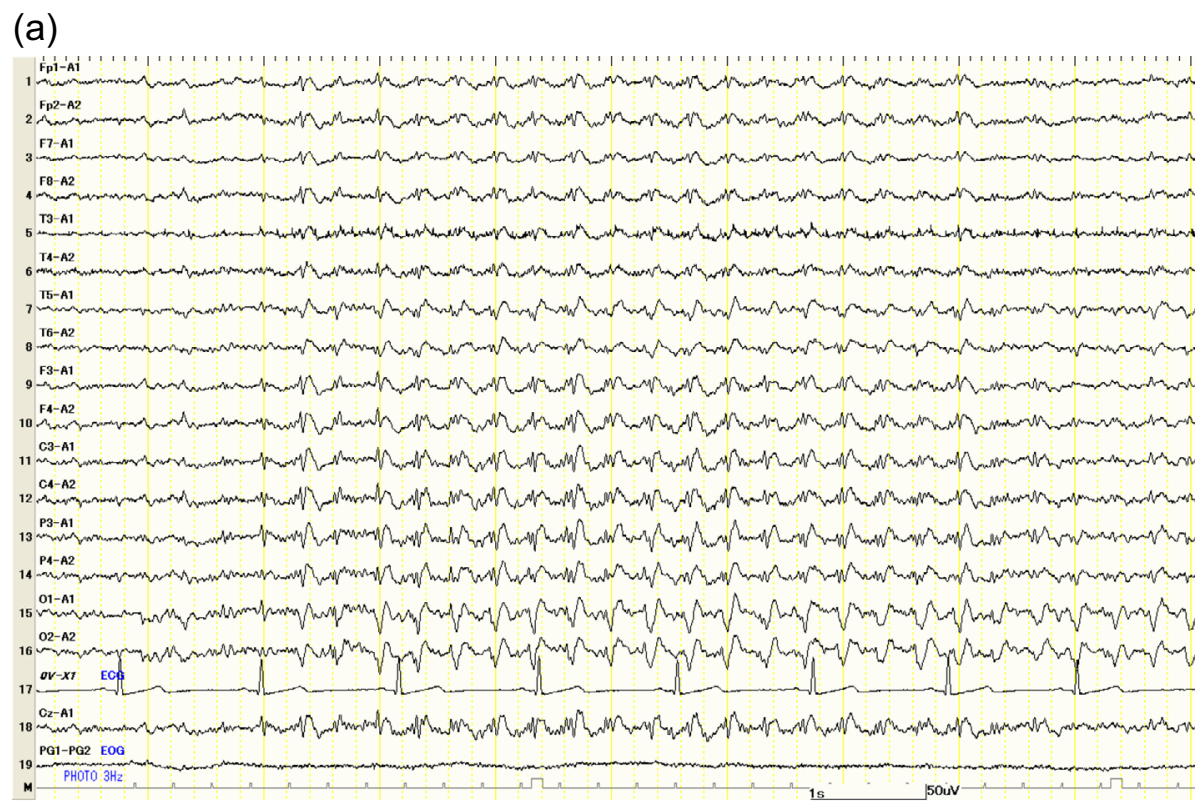

(b)

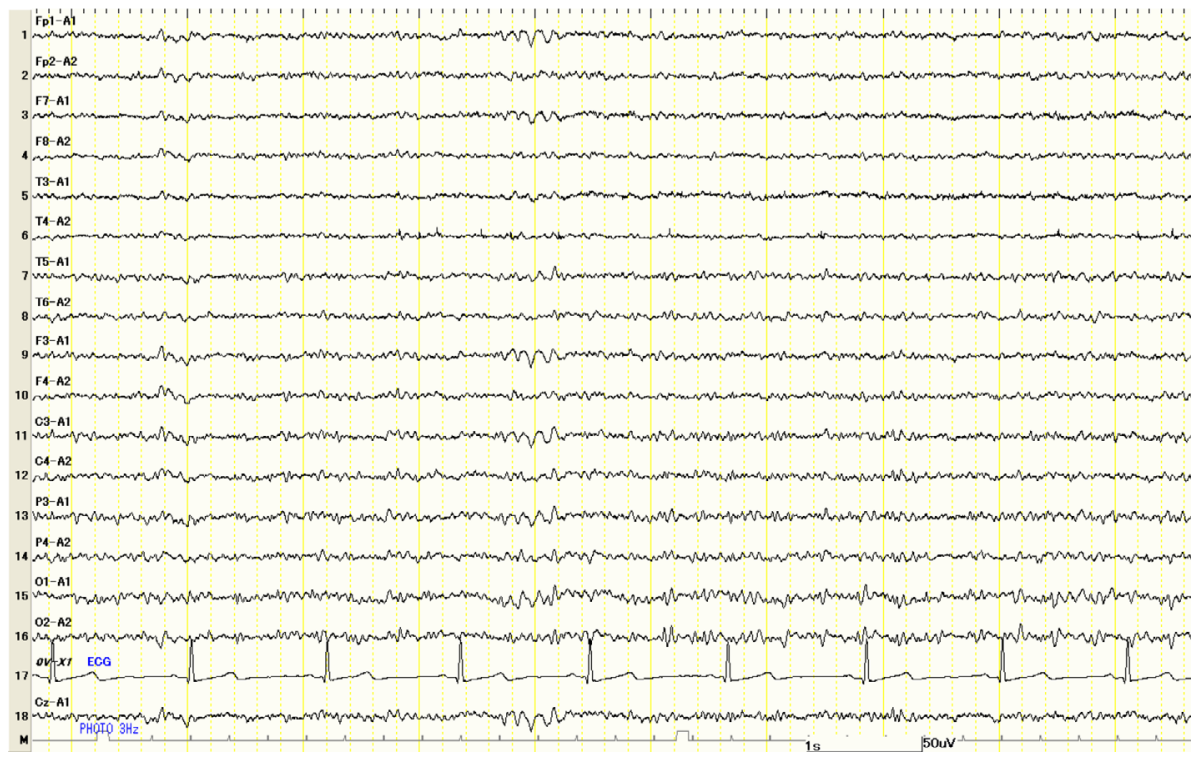

Figure 2. Electroencephalography of Patient 2. (a) Occipital dominant spike-wave complexes were observed with 3-Hz photic stimulation before treatment. (b) Occipital dominant spike-wave complexes were decreased four weeks after medication with KRB.

value, 0.65 to 1.07$)$ and estimated glomerular filtration rate of $41 \mathrm{~mL} / \mathrm{min} / 1.73 \mathrm{~m}^{2}$. After three days of treatment, he slept well throughout the night and no longer suffered from abnormal sleep behaviors. He continued to take KRB for two years, and the abnormal sleep behaviors never recurred, even though he sometimes forgot to take KRB.

\section{Discussion}

KRB was originally described in the ancient Chinese medical text Jin Gui Yao Lue written in the 2nd century AD. In the text, KRB was described as effective for treating a declining sexual desire, sexual over-fatigue, a hypertonic abdominal rectus muscle and cold genitalia, diarrhea, anemia, feeling dizzy, alopecia, and an extremely weak pulse (12).
Men often experienced nocturnal ejaculation, while women experienced sexually aroused dreams. In traditional Japanese medicine, for hundreds of years, such abnormal nocturnal sexual behaviors had been treated with KRB. As sleeprelated abnormal sexual behaviors are related to parasomnia, we tried to expand the indications for this drug to a broader range of abnormal sleep-related behaviors.

In our case-series, Patient 1 was diagnosed with RBD and Patient 3 with probable RBD. Patient 2 displayed a parasomnia overlap disorder in which features of both probable RBD and arousal disorders were observed $(17,18)$. Sleepwalking is generally considered as NREM parasomnia and is most commonly seen in young adults. However, a recent study demonstrated that $0.9 \%$ of elderly men (mean age, 75.6 years old) had probable sleep walking with or without 
RBD (19). A definite diagnosis of abnormal behaviors during sleep in Patient 4 was difficult, since we were unable to obtain vPSG data. The differential diagnosis of parasomnia includes hypoglycemia and epilepsy (20). Patients with insulinoma who present with abnormal nocturnal behavior and injury during sleep, resembling $\mathrm{RBD}$, have been reported $(21,22)$. However, we detected no pancreatic tumor, and the patient reported that he had never experienced a hypoglycemic attack. His abnormal behavior in sleep has not recurred for two years despite no longer taking anti-epileptic drugs, such as carbamazepine, suggesting that the presence of epilepsy was unlikely even though we could not rule out this diagnosis completely.

More than $1 \%$ of the population $\geq 60$ years old have a parasomnia (17). Importantly, RBD is a strong prodromal marker of synucleinopathy. In a recent study of $1280 \mathrm{vPSG}-$ confirmed idiopathic RBD patients, $6.25 \%$ pheno-converted to dementia or parkinsonism per year (23). Postural instability is a common feature in these neurodegenerative diseases. Medications, such as clonazepam, used for RBD often exacerbate the tendency to fall. Thus, it is noteworthy that KRB was effective for RBD.

The mechanism underlying the pharmacological activity of KRB remains to be elucidated. However, the observed reduction in occipital dominant spike-wave complexes induced by $3-\mathrm{Hz}$ photic stimulation after KRB treatment in Patient 2 suggests that KRB has inhibitory effects on brain irritability. In addition, Saikokaryukotsuboreitou (SRB), a closely related herbal medicine, shares five common crude drugs (cinnamon bark, jujube, oyster shell, longgu, glycyrrhiza, and ginger) with $\mathrm{KRB}$ and has been used to treat neurological symptoms (24), indicating that these drugs exert effects on the brain. Traditionally, both KRB and SRB have been prescribed for parasomnia for hundreds of years. Nocturnal abnormal sexual behaviors, now classified as confusional arousals, have been treated with KRB, and KRB has been considered have milder effects and be more suitable for elderly patients than other agents.

Recently, another herbal medicine, YKS, was reported to be effective for treating idiopathic RBD (25). In preclinical studies, YKS has been shown to regulate neurotransmitters, including GABA, serotonin, glutamate, and dopamine; regulate the expression of dynamin and glutamate transporters; and regulate the levels of glucocorticoid hormones and related enzymes, such as choline acetyltransferase and acetylcholinesterase (26). YKS consists of seven crude drugs, none of which are shared by KRB. Thus, YKS and KRB work through different mechanisms of action. In traditional Japanese medicine, YKS has mainly been prescribed for treating crying at night in children. Recently, its indication was extended to the treatment of behavioral and psychological symptoms of dementia (BPSD). Compared with YKS, $\mathrm{KRB}$ can be used for patients with a more greatly reduced physical strength level (12). Further studies are necessary to compare the efficacy of YKS and KRB for the treatment of parasomnia.
No adverse reactions were observed in our patients during at least a two-year observation period, and KRB is generally considered safe. However, patients who are prescribed other glycyrrhiza-containing drugs in addition to KRB should be carefully monitored for pseudoaldosteronism, which may manifest as hypokalemia, edema, increased blood pressure, and myopathy.

Several limitations associated with the present study warrant mention. First, we included a limited number of patients with heterogeneous abnormal behaviors in sleep. Second, we were able to obtain vPSG findings in only one patient and electroencephalography findings in two patients. Since all four patients started medication with KRB soon after their first visit to our clinic and their sleep-related symptoms were alleviated within a week, they did not wish to undergo further examinations. Therefore, we made a provisional diagnosis of probable RBD in Patients 2 and 3. Which type of parasomnia is most effectively treated with $\mathrm{KRB}$ remains unclear.

In conclusion, $\mathrm{KRB}$, a traditional Japanese herbal medicine, may be beneficial for the treatment of parasomnias in the elderly.

\section{The authors state that they have no Conflict of Interest (COI).}

\section{References}

1. Bornemann MC, Schenck C, Rosen G, Pressman M. Parasomonias. In: International Classification of Sleep Disorders. 3rd ed. Sateia M, Ed. American Academy of Sleep Medicine, 2014: 225280.

2. Mahowald MW, Bornemann MC, Schenck CH. Parasomnias. Semin Neurol 24: 283-292, 2004.

3. Mahowald MW, Schenck $\mathrm{CH}$. Insights from studying human sleep disorders. Nature 437: 1279-1285, 2005.

4. Tinuper P, Bisulli F, Provini F. The parasomnias: mechanisms and treatment. Epilepsia 53 (Suppl 7): 12-19, 2012.

5. Barone DA, Henchcliffe C. Rapid eye movement sleep behavior disorder and the link to alpha-synucleinopathies. Clin Neurophysiol 129: 1551-1564, 2018.

6. Mahowald MW, Schenck CH. REM sleep behaviour disorder: a marker of synucleinopathy. Lancet Neurol 12: 417-419, 2013.

7. Schenck CH, Bundlie SR, Mahowald MW. Delayed emergence of a parkinsonian disorder in $38 \%$ of 29 older men initially diagnosed with idiopathic rapid eye movement sleep behaviour disorder. Neurology 46: 388-393, 1996.

8. Iranzo A, Molinuevo JL, Santamaria J, et al. Rapid-eye-movement sleep behaviour disorder as an early marker for a neurodegenerative disorder: a descriptive study. Lancet Neurol 5: 572-577, 2006.

9. Hogl B, Stefani A, Videnovic A. Idiopathic REM sleep behaviour disorder and neurodegeneration - an update. Nat Rev Neurol 14: 40-55, 2018.

10. Castelnovo A, Lopez R, Proserpio P, Nobili L, Dauvilliers Y. NREM sleep parasomnias as disorders of sleep-state dissociation. Nat Rev Neurol 14: 470-481, 2018.

11. Gagnon JF, Postuma RB, Mazza S, Doyon J, Montplaisir J. Rapideye-movement sleep behaviour disorder and neurodegenerative diseases. Lancet Neurol 5: 424-432, 2006.

12. Tahara E. Dementia - Abnormal Behavior. In: Textbook of Traditional Japanese Medicine. Arai M, Hioki C, Kosoto H, Yoshikawa M, Muramatsu S, Katai S, et al., Eds. 2017:169-172 [Internet]. 
Available from: http://kampotextbook.sakura.ne.jp/

13. Ministry of Health, Labor and Welfare. The Japanese Pharmacopeia [Internet]. Available from: https://www.mhlw.go.jp/stf/seisak unitsuite/bunya/0000066597.html.

14. Asari S, Fujimoto K, Miyauchi A, Sato T, Nakano I, Muramatsu S. Subregional 6- $\left[{ }^{18} \mathrm{~F}\right]$ fluoro-L-m-tyrosine uptake in the striatum in Parkinson's disease. BMC Neurol 11: 35, 2011.

15. Boeve BF. Idiopathic REM sleep behaviour disorder in the development of Parkinson's disease. Lancet Neurol 12: 469-482, 2013.

16. Postuma RB. Diagnosing REM sleep behavior disorder in Parkinson's disease-can we avoid the polysomnogram? Mov Disord 29: 713-714, 2014.

17. Sateia MJ. International classification of sleep disorders-third edition: highlights and modifications. Chest 146: 1387-1394, 2014.

18. Bargiotas P, Muellner J, Schuepbach WMM, Bassetti CL. Parasomnia overlap disorder, Parkinson's disease and subthalamic deep brain stimulation: three case reports. BMC Neurol 17: 137, 2017.

19. Zhang X, Molsberry SA, Pavlova M, Schwarzschild MA, Ascherio A, Gao X. Association of Sleepwalking and REM Sleep Behavior Disorder With Parkinson Disease in Men. JAMA Netw Open 4: e 215713, 2021.

20. Antelmi E, Lippolis M, Biscarini F, Tinazzi M, Plazzi G. REM sleep behavior disorder: Mimics and variants. Sleep Med Rev 60: 101515, 2021.

21. Kishi K, Kubo K, Tomita T, Nakamura K, Yasui-Furukori N. Insu- linoma resembling a rapid eye movement sleep behavior disorder: a case report. Int Med Case Rep J 12: 51-54, 2019.

22. Suzuki K, Kawasaki A, Miyamoto M, et al. Insulinoma Masquerading as Rapid Eye Movement Sleep Behavior Disorder: Case Series and Literature Review. Medicine (Baltimore) 94: e1065, 2015.

23. Postuma RB, Iranzo $\mathrm{A}, \mathrm{Hu} \mathrm{M}$, et al. Risk and predictors of dementia and parkinsonism in idiopathic REM sleep behaviour disorder: a multicentre study. Brain 142: 744-759, 2019.

24. Kwon CY, Lee B, Chung SY, et al. Efficacy and safety of Sihogayonggolmoryeo-tang (Saikokaryukotsuboreito, Chai-Hu-JiaLong-Gu-Mu-Li-Tang) for post-stroke depression: A systematic review and meta-analysis. Sci Rep 9: 14536, 2019.

25. Matsui K, Sasai-Sakuma T, Ishigooka J, Nishimura K, Inoue Y. Effect of Yokukansan for the Treatment of Idiopathic Rapid Eye Movement Sleep Behavior Disorder: A Retrospective Analysis of Consecutive Patients. J Clin Sleep Med 15: 1173-1178, 2019.

26. Jang JH, Jung K, Kim JS, Jung I, Yoo H, Moon C. Potential Application of Yokukansan as a Remedy for Parkinson's Disease. Evid Based Complement Alternat Med 2018: 1875928, 2018.

The Internal Medicine is an Open Access journal distributed under the Creative Commons Attribution-NonCommercial-NoDerivatives 4.0 International License. To view the details of this license, please visit (https://creativecommons.org/licenses/ by-nc-nd/4.0/).

(C) The Japanese Society of Internal Medicine Intern Med Advance Publication 